\title{
Systematische Untersuchungen zur Funktionsweise des Kantenabsorbers als "Modenbremse"
}

E. Kurz(D, G. Graber, W. Weselak

Das heutige Wissen über den akustischen Einfluss von Kantenabsorbern auf ein Schallfeld basiert hauptsächlich auf empirischen Erfahrungen. Da es kein rechnerisches Modell für Kantenabsorber gibt, wird diese Art von Absorbern leider nur zögerlich in der raumakustischen Planung eingesetzt.

Dieser Beitrag untersucht das Absorptionsverhalten von Kantenabsorbern systematisch und schlägt einen Ansatz für ein rechnerisches Modell für Kantenabsorber in einfachen Raumgeometrien vor. Für die systematischen Untersuchungen wurde ein modularer Kantenabsorber entwickelt und konstruiert. Im Entwicklungsprozess wurden optimale Parameter für den Strömungswiderstand, das Perforationsverhältnis des Absorbergehäuses und die Schichtdicke des eingebauten porösen Materials gefunden. Durch das Baukastenprinzip des entwickelten Kantenabsorbers ist ein flexibler Aufbau möglich.

Es wurden verschiedene Konfigurationen des modularen Kantenabsorbers im Hallraum gemessen. Das akustische Verhalten des Kantenabsorbers wurde in mehreren Hörsälen ermittelt. Es konnte festgestellt werden, dass die äquivalente Absorptionsfläche pro Längeneinheit sowie die untere Grenzfrequenz des Kantenabsorbers abhängig von der Einbautiefe und der jeweiligen Montagekante des Kantenabsorbers sind. Außerdem ist die Bandbreite für die effektive Absorption von den Abmessungen des Kantenabsorbers abhängig.

Der modulare Kantenabsorber wurde bei einer raumakustischen Sanierung eingesetzt und Vorher-Nachher-Messungen wurden durchgeführt, um die Effektivität des Kantenabsorbers zu bestätigen.

Schlüsselwörter: Raumakustik; Kantenabsorber; Hallraum; modales Schallfeld

Systematic investigations of the functional principle of the edge absorber as a "modal brake".

Today's knowledge about the acoustic influence of edge absorbers on a sound field is mainly based on empirical experience. Unfortunately, since there is no computational model for edge absorbers, this type of absorber is only hesitantly used in room acoustic design.

This contribution systematically investigates the absorption behavior of edge absorbers and proposes an approach for a computational model for edge absorbers in simple room geometries. A modular edge absorber was developed and constructed for systematic investigations. In the development process, optimal parameters for flow resistance, the perforation ratio of the absorber housing and the layer thickness of the incorporated porous material were found. Due to the modular design principle of the developed edge absorber, a flexible assembly is possible.

Various configurations of the modular edge absorber were measured in the reverberation chamber. The acoustic behavior of the edge absorber was determined in several lecture halls. It was found that the equivalent absorption area per unit of length and the lower frequency limit of the edge absorber are dependent on the assembly depth and the particular mounting edge of the edge absorber. In addition, the bandwidth for effective absorption depends on the dimensions of the edge absorber.

The modular edge absorber was used in a room acoustic renovation and pre-post measurements were performed to confirm the effectiveness of the edge absorber.

Keywords: room acoustics; edge absorber; reverberation chamber; modal sound field

Eingegangen am 22. Jänner 2021, angenommen am 11. März 2021, online publiziert am 25. März 2021

\section{Einleitung}

Sowohl die Verständlichkeit von Sprache als auch die Transparenz von Musik in einem Raum sind stark von dessen akustischen Eigenschaften abhängig. Wenn die Sprachverständlichkeit in einem Raum schlecht ist, müssen zusätzliche kognitive Ressourcen zur Verfügung gestellt werden, um die gesprochene Information zu verarbeiten. Daher ist eine adäquate akustische Behandlung von Räumen notwendig und wird oft durch den Einbau einer absorbierenden Oberflächenverkleidung aus faserigen oder porösen Materialien erreicht. Mit diesen Absorbern ist die effektive Schallfeldkontrolle für Frequenzen oberhalb von $250 \mathrm{~Hz}$ gegeben. Die wirksame Schall- feldkontrolle für tiefe Frequenzen ist eine anspruchsvollere, jedoch umso wichtigere Aufgabe.

Aus psychoakustischen Untersuchungen von Wegel [1] und Tobias [2] wissen wir um den breitbandigen Maskierungseffekt von tiefen Frequenzen, insbesondere für Frequenzen oberhalb der Mas-

Kurz, Eric, Institut für Signalverarbeitung und Sprachkommunikation, Technische Universität Graz, Inffeldgasse 16c/EG, 8010 Graz, Österreich (E-Mail: eric.kurz@tugraz.at); Graber, Gerhard, Institut für Signalverarbeitung und Sprachkommunikation, Technische Universität Graz, Graz, Österreich; Weselak, Werner, Institut für Signalverarbeitung und Sprachkommunikation, Technische Universität Graz, Graz, Österreich 

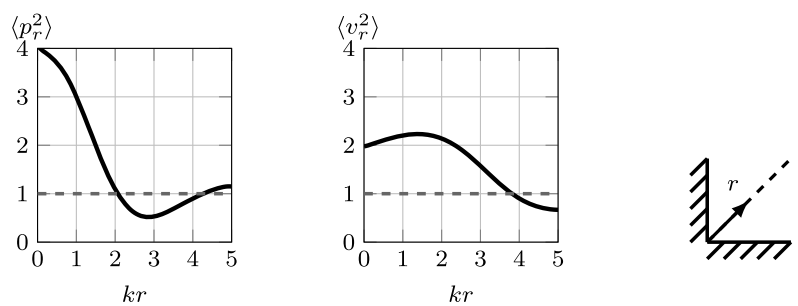

Abb. 1. Relative potentielle Energie $\left\langle p_{r}^{2}\right\rangle$ und kinetische Energie $\left\langle v_{r}^{2}\right\rangle$ vor einer Kante, die in einem Quadranten eines offenen Raums liegt, gemittelt über alle Einfallsrichtungen [8]

kierungsfrequenz $f_{M}$ (vgl. [3]). Außerdem konnte die Bedeutung der tieffrequenten Schallfeldkontrolle in der Raumakustik und deren Auswirkung auf die Sprachverständlichkeit und die Transparenz von Musik durch Untersuchungen von Kuttruff [4, p. 617] und Fuchs [5-7] gezeigt werden. Um das tieffrequente Schallfeld zu kontrollieren, ist es sehr wichtig auf die Platzierung von absorbierendem Material im Raum zu achten und dessen Wechselwirkung mit dem Schallfeld zu untersuchen. Für tiefe Frequenzen bestimmen die modalen Eigenschaften, die hauptsächlich von den Resonanzfrequenzen des Raumes beeinflusst werden, die Verteilung des Schalldrucks $p$ und der Schallschnelle $v$ im Raum. Somit kann diese Verteilung genutzt werden, um die optimalen Positionen für absorbierendes Material in einem Raum zu bestimmen. Außerdem geht aus theoretischen Untersuchungen von Waterhouse [8] hervor, dass insbesondere in Ecken und Kanten eines Raumes hohe Schalldrücke und -schnellen zu erwarten sind. Aufgrund der Spiegelquellentheorie verursacht eine eintreffende planare Schallwelle aus einem offenen Viertelraum in einer Kante konstruktive Interferenzen und somit eine Erhöhung der relativen potentiellen und kinetischen Energie vor dieser Kante (vgl. Abb. 1).

Basierend auf diesem Wissen ist es verständlich, dass Kantenabsorber (KA), auch Bassfallen genannt, als tieffrequente Schalldämpfer bekannt und dementsprechend in der Raumakustik sehr beliebt sind. Die Vorteile von KA liegen auf der Hand. Sie bieten eine breitbandige Absorption für tiefe Frequenzen (ca. $30 \mathrm{~Hz}$ bis $250 \mathrm{~Hz}$ ) und können mit einem ausgewogenen Preis-Leistungs-Verhältnis hergestellt werden. Bei raumakustischen Sanierungen kann die nachträgliche Montage von KA oft mit geringem Aufwand erfolgen und ihr subtiles architektonisches Konzept erlaubt eine unauffällige Integration in bestehende Räumlichkeiten (z.B. als Abdeckung für Leitungen oder als Sitzbänke).

Jedoch gibt es kein hinreichendes Simulationswerkzeug für den Einsatz von KA in der Planung raumakustischer Sanierungen. Auf der einen Seite ist die akustische Simulation, welche Spiegelquellen und Raytracing verwendet (z.B. [9]), nicht für die Simulation von KA geeignet, da sie für tiefe Frequenzen nicht hinreichend genau ist. Auf der anderen Seite sind Berechnungen mit FEMbasierter Software wie z. B. COMSOL [10] insbesondere für komplexere Schallfelder sehr zeitaufwendig und daher unpraktisch für den raumakustischen Planungsprozess. Daher besteht offensichtlich ein Bedarf an einer geeigneten und rechnerisch effektiven KASimulationssoftware.

Dieser Beitrag versucht ein detaillierteres Verständnis der Prinzipien der modalen Schallfeldsteuerung mit Hilfe von KA zu entwickeln und schlägt geeignete akustische Parameter für eine Simulation von $\mathrm{KA}$ vor. Die in dieser Arbeit vorgestellten Untersuchungen basieren auf Teilen der Masterarbeit von D. Reisinger [11] und ergänzen Untersuchungen aus [12]. Die Arbeit ist folgendermaßen aufgebaut: Der zweite Abschnitt gibt einen kurzen Überblick über die grundlegenden Absorptionsprinzipien von KA. Abschnitt 3 beschreibt den entwickelten $K A$, der in unseren Untersuchungen eingesetzt wurde, und zeigt den Aufbau für die Messungen, die sowohl im Hallraum als auch in verschiedenen Hörsälen durchgeführt wurden. Um ein Verständnis für das akustische Verhalten des KA zu bekommen, werden zunächst die Ergebnisse der Messungen im Hallraum vorgestellt (Abschnitt 4.1). Abschnitt 4.2 beschreibt die Ergebnisse der Messungen, die in verschiedenen Hörsälen durchgeführt wurden. Eine Diskussion sowie eine kurze Zusammenfassung der gewonnenen Erkenntnisse runden den Beitrag ab.

\section{Theoretische Untersuchungen}

Vereinfacht kann ein KA als passiver Absorber betrachtet werden, dessen Absorptionswirkung auf der Wechselwirkung zwischen porösem Absorber und Modalfeld in Raumkanten basiert. Er wird, wie der Name vermuten lässt, in den Kanten und auch Ecken eines Raumes eingebracht. In früheren Untersuchungen von Maa [13] und Kuhl [14] konnte gezeigt werden, dass der Einsatz von porösem Absorptionsmaterial in Raumkanten und -ecken die effektive Absorption, insbesondere im unteren Frequenzbereich, erhöht. Ein moderner KA besteht aus einer Schachtkonstruktion, die mit porösem Absorbermaterial gefültt ist. Die Höhe und die Tiefe des Kanals sind gering im Vergleich zu seiner Länge.

\subsection{Interferenzphänomen}

Das Hauptabsorptionsprinzip des KA kann durch die Betrachtung von Interferenzphänomenen in der Nähe einer akustisch starren Kante und das modale Schallfeld in einem Raum erklärt werden.

In einem ersten Schritt gehen wir davon aus, dass die $x-y-$ und $x-$ $z$-Ebene eine akustisch harte Kante ( $x$-Achse) in einem unendlichen offenen Viertelraum bilden. Die Kante ist groß im Vergleich zur Wellenlänge einer sinusförmigen ebenen Schallwelle mit Einheitsamplitude, die aus dem Unendlichen mit den Einfallswinkeln $\vartheta$ und $\varphi$ einfällt (vgl. Abb. 2, rechts). Für diese Anordnung kann ein Spiegelquellenmodell angenommen werden. Durch die Überlagerung der einfallenden und der reflektierten Welle sind Interferenzeffekte bei Schalldruck $p$ und Schallschnelle $v$ zu erwarten. Beide Größen variieren in Abhängigkeit von der Entfernung zur Kante und dem Einfallswinkel der Schallwelle. Es kann gezeigt werden (vgl. [8]), dass für dieses Modell folgende Proportionalitäten für die relative potentielle Energie $\left\langle p^{2}\right\rangle$ und die relative kinetische Energie $\left\langle v^{2}\right\rangle$ gültig sind:

$$
\begin{aligned}
\left\langle p^{2}\right\rangle & \sim 4(\cos b \cos c)^{2}, \\
\left\langle v^{2}\right\rangle & =\left\langle v_{x}^{2}\right\rangle+\left\langle v_{y}^{2}\right\rangle+\left\langle v_{z}^{2}\right\rangle \text { mit } \\
\left\langle v_{x}^{2}\right\rangle & \sim 4(\cos \alpha \cos b \cos c)^{2}, \\
\left\langle v_{y}^{2}\right\rangle & \sim 4(\cos \beta \sin b \cos c)^{2} \text { und } \\
\left\langle v_{z}^{2}\right\rangle & \sim 4(\cos \gamma \cos b \sin c)^{2} .
\end{aligned}
$$

Hierbei sind die einzelnen Terme wie folgt definiert:

$$
\begin{aligned}
\cos \alpha & =\cos \vartheta, \\
\cos \beta & =\sin \vartheta \cos \varphi, \\
\cos \gamma & =\sin \vartheta \sin \varphi, \\
b & =k y \cos \beta, \\
c & =k z \cos \gamma .
\end{aligned}
$$

Abbildung 2 zeigt das Verhalten der relativen potentiellen sowie kinetischen Energie einer einzelnen ebenen Welle für $k r=1$ in Abhängigkeit von den Einfallswinkeln $\vartheta$ und $\varphi$. Aufgrund der Symmetrie 

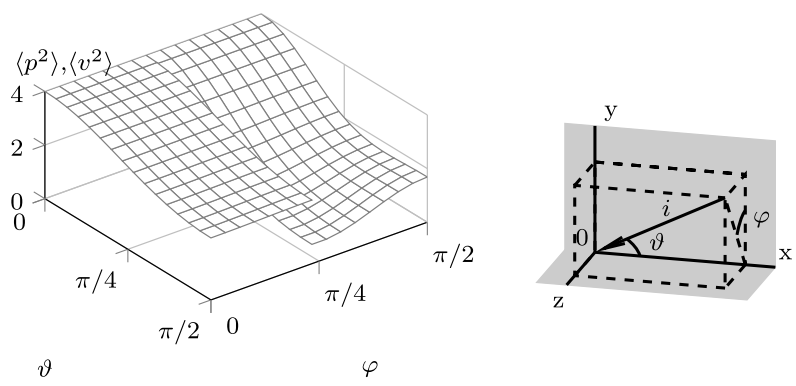

Abb. 2. Relative potentielle und kinetische Energie $\left(\left\langle p^{2}\right\rangle,\left\langle v^{2}\right\rangle\right)$ einer einzelnen ebenen Welle $k r=1$ in Abhängigkeit der Einfallswinkel $\vartheta$ und $\varphi$ (links). Angenommenes geometrisches Modell mit spiegelnden $x-y$ - und $x-z-E b e n e n$ und einer ebenen Welle $i$ mit Einfallswinkeln $\vartheta$ und $\varphi$ (rechts)

sind die abhängigen Flächen für $\left\langle p^{2}\right\rangle$ und $\left\langle v^{2}\right\rangle$ für $0 \leq \varphi \leq \pi / 4$ bzw. $\pi / 4 \leq \varphi \leq \pi / 2$ eingezeichnet.

Die Ergebnisse für $\left\langle p^{2}\right\rangle$ und $\left\langle v^{2}\right\rangle$ zeigen leichte Unterschiede. Man sieht, dass für einen streifenden Einfallswinkel $\vartheta \rightarrow 0\left\langle p^{2}\right\rangle \approx\left\langle v^{2}\right\rangle$ gilt. Bei senkrechtem Schalleinfall in der Symmetrieebene der Kante $(\vartheta=\pi / 2, \varphi=\pi / 4)$ ist $\left\langle p^{2}\right\rangle=2,37$ und $\left\langle v^{2}\right\rangle=0,72$. Streift die Schallwelle die $x-y$ - bzw. $x$-z-Ebene $(\varphi \rightarrow 0$ resp. $\pi / 2)$ wird eine mittlere Verstärkung von $\overline{\left\langle p^{2}\right\rangle}=3,12$ und $\overline{\left\langle v^{2}\right\rangle}=2,45$ erreicht.

Die Integration über alle möglichen Einfallswinkel $\vartheta$ und $\varphi$ der Viertelkugel erfolgt durch

$$
\left\langle p_{r}^{2}\right\rangle=\frac{1}{\pi} \int_{0}^{\pi} \int_{0}^{\pi / 2}\left\langle p^{2}\right\rangle \sin (\vartheta) d \varphi d \vartheta
$$

und

$$
\left\langle v_{r}^{2}\right\rangle=\frac{1}{\pi} \int_{0}^{\pi} \int_{0}^{\pi / 2}\left\langle v^{2}\right\rangle \sin (\vartheta) d \varphi d \vartheta
$$

Das Einsetzen von (1) und (2) in (3) und (4) führt zu den Ausdrücken

$$
\left\langle p_{r}^{2}\right\rangle \sim 1+j_{0}(2 k y)+j_{0}(2 k z)+j_{0}(2 k r)
$$

und

$$
\begin{aligned}
\left\langle v_{r}^{2}\right\rangle \sim 1 & -j_{0}(2 k y)+2 \frac{j_{1}(2 k y)}{k y} \\
& -j_{0}(2 k z)+2 \frac{j_{1}(2 k z)}{k z} \\
& -j_{0}(2 k r)+2 \frac{j_{1}(2 k r)}{k r} .
\end{aligned}
$$

Hierbei ist $r^{2}=y^{2}+z^{2}$ und $j_{n}(x)$ ist die sphärische Besselfunktion

$$
j_{n}(x)=(-x)^{n}\left(\frac{1}{x} \frac{d}{d x}\right)^{n} \frac{\sin x}{x} .
$$

Wir können (5) und (6) auf der Symmetrieebene der Kante $(\varphi=\pi / 4$ bzw. $y=z$ ) mit Bezug auf den Abstand $r$ und die Frequenz $f$ der einfallenden ebenen Schallwellen auswerten. Die Ergebnisse dieser Auswertung sind in den Abbildungen 3 und 4 dargestellt. Für die beiden relativen Energien $\left\langle p_{r}^{2}\right\rangle$ und $\left\langle v_{r}^{2}\right\rangle$ lassen sich zwei Bereiche unterscheiden. Der erste Bereich wird von konstruktiven Interferenzen dominiert, die zu hohen relativen Energien $\left\langle p_{r}^{2}\right\rangle \approx 4$ und $\left\langle v_{r}^{2}\right\rangle \approx 2$ führen. Für ein größeres $r$ und höhere Frequenzen $f$ ist diese konstruktive Interferenz nicht mehr gegeben. $\left\langle p_{r}^{2}\right\rangle$ sinkt auf einen Mittelwert $\approx 1$ mit einer Welligkeit in Abhängigkeit von $r$ und $f$. Dasselbe gilt für $\left\langle v_{r}^{2}\right\rangle$, allerdings mit einer etwas stärkeren Welligkeit.

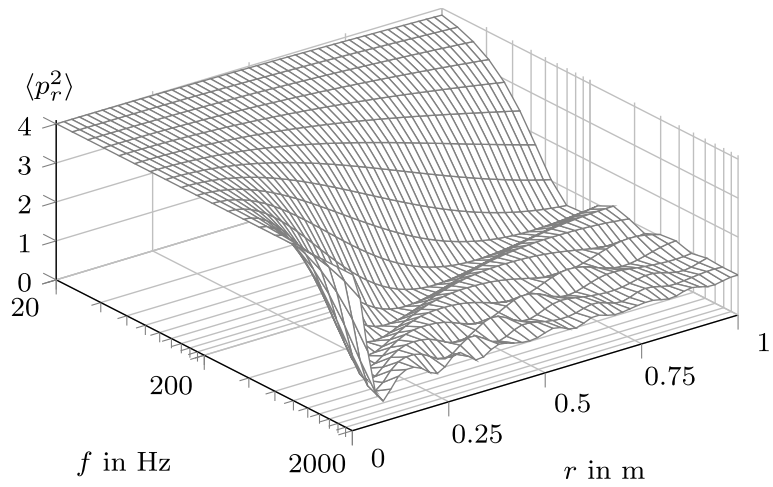

Abb. 3. Mittlere potentielle Energie $\left\langle p_{r}^{2}\right\rangle$ entlang der Symmetrieebene der Kante $(\varphi=\pi / 4)$ in Abhängigkeit von der Entfernung $r$ und der Frequenz $f$ der einfallenden ebenen Wellen

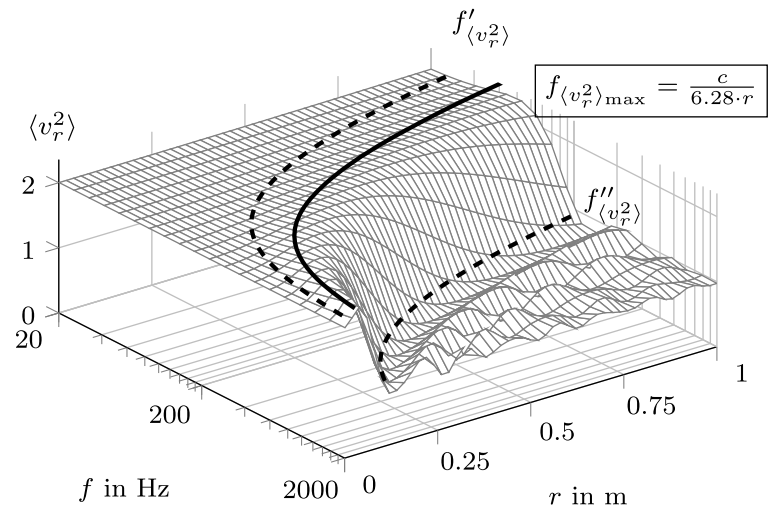

Abb. 4. Mittlere kinetische Energie $\left\langle v_{r}^{2}\right\rangle$ entlang der Symmetrieebene der Kante $(\varphi=\pi / 4)$ in Abhängigkeit von der Entfernung $r$ und der Frequenz $f$ der einfallenden ebenen Wellen. - : Angepasste Funktion für das frequenzabhängige Verhalten der maximalen mittleren kinetischen Energie $\left\langle v_{r}^{2}\right\rangle_{\max }$ - - -: Abstandsabhängige untere und obere Grenzfrequenzen $f_{\left\langle v_{r}^{2}\right\rangle}^{\prime}$ und $f_{\left\langle v_{r}^{2}\right\rangle}^{\prime \prime}$

Eine genauere Analyse von Abb. 4 zeigt einen deutlichen Abfall von $\left\langle v_{r}^{2}\right\rangle$, der die beiden Bereiche trennt, sowie ein Maximum von $\left\langle v_{r}^{2}\right\rangle_{\max } \approx 2,2$. Außerdem scheint das Verhalten von $\left\langle v_{r}^{2}\right\rangle$ für kleine $r$ zunächst unerklärlich zu sein, wenn man annimmt, dass die Schallschnelle in der Nähe einer akustisch harten Oberfläche gegen Null gehen muss. Dieser Effekt gilt jedoch hauptsächlich für Schallschnellekomponenten orthogonal zur akustisch harten Oberfläche. Vernachlässigt man die akustische Grenzschicht an der Oberfläche, so müssen die streifenden Komponenten von $v$ jedoch nicht abnehmen. Daher dominieren sie das Verhalten von $\left\langle v_{r}^{2}\right\rangle$. Auch das flache und frequenzunabhängige Verhalten von $\left\langle v_{r}^{2}\right\rangle$ für den ersten Bereich kann mit diesem Wissen erklärt werden.

Geht man nun beispielsweise davon aus, dass $\left\langle v_{r}^{2}\right\rangle_{\max }$ den Rand des Bereichs mit $\left\langle v_{r}^{2}\right\rangle \approx 2$ markiert, so kann eine Grenzfrequenz $f_{\left\langle v_{r}^{2}\right\rangle_{\max }}$ in Abhängigkeit von $r$ definiert werden. Eine Anpassung mit den $\left\langle v_{r}^{2}\right\rangle_{\max }$-Daten und mit einem Modell ähnlich der $\frac{\lambda}{4}$-Regel wurde durchgeführt, um den Parameter $\psi_{\max }$ (8) zu finden. Für $f_{\left\langle v_{r}^{2}\right\rangle_{\max }}$ kann eine hohe kinetische Energie des Schallfeldes im Abstand $r$ vor der Raumkante erwartet werden. Weiterhin können abstandsabhängige obere und untere Grenzfrequenzen $\left(f_{\left\langle v_{r}^{2}\right\rangle}^{\prime \prime}\right.$ und $\left.f_{\left\langle v_{r}^{2}\right\rangle}^{\prime}\right)$ definiert werden (9), (10).

$$
f_{\left\langle\left\langle v_{r}^{2}\right\rangle_{\max }\right.}=\frac{c}{\psi_{\max } \cdot r}=\frac{c}{2 \pi \cdot r} \quad \text { bzw. } \quad k r=1
$$




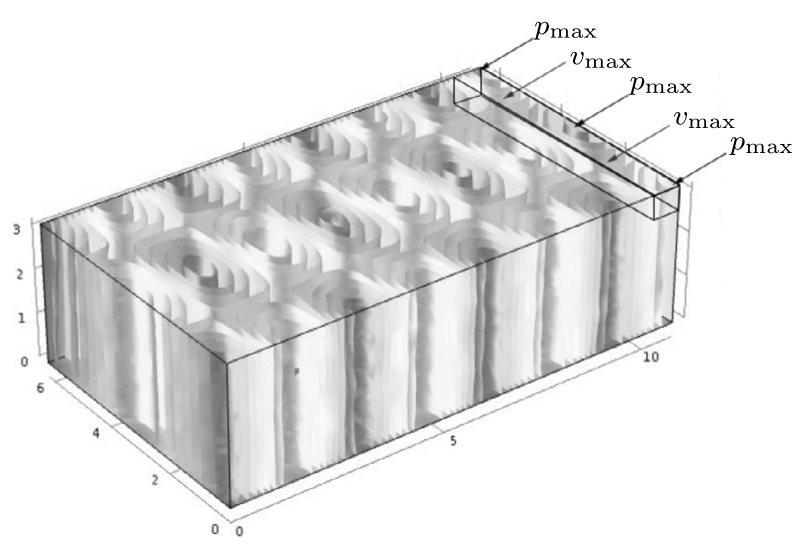

Abb. 5. Simulation eines modalen Schallfeldes mit der Modenordnung 7-2-0 für eine einfache Raumgeometrie mit den Abmessungen $L \times B \times H: 10.8 \times 6.24 \times 3.15 \mathrm{~m}[15]$

$$
\begin{array}{ll}
f_{\left\langle v_{r}^{2}\right\rangle}^{\prime \prime}=\frac{c}{\pi / 2 \cdot r} \quad \text { bzw. } & k r=4 \\
f_{\left\langle v_{r}^{2}\right\rangle}^{\prime}=\frac{c}{4 \pi \cdot r} \quad \text { bzw. } \quad k r=\frac{1}{2}
\end{array}
$$

Die Ergebnisse dieser theoretischen Betrachtung führen zu dem Eindruck, dass die Führung des Schallfeldes durch die akustisch harte Kante für eine parallel zur Kante verlaufende Zunahme von Schalldruck und Schallschnelle verantwortlich ist. Besonders im Frequenzbereich $f_{\left\langle v_{r}^{2}\right\rangle}^{\prime}<f<f_{\left\langle v_{r}^{2}\right\rangle}^{\prime \prime}$ ist eine hohe Schallenergie zu erwarten.

\subsection{Modales Schallfeld}

In einem nächsten Schritt gehen wir von einer niederfrequenten Anregung im Raum aus, welche zu einem modalen Schallfeld führt. Dieses modale Schallfeld und damit die Verteilung der Schalldruckund Schallschnellemaxima wird hauptsächlich durch die Raumgeometrie bestimmt. Diese Maxima treten besonders in den Kanten und Ecken eines Raumes auf, wo zwei Grenzflächen die Wellenausbreitung, parallel zur Raumkante, führen (vgl. Abb. 5).

Der in Abschnitt 2.1 dargestellte Gewinn an potentieller und kinetischer Energie führt durch die stehenden Wellen parallel zu einer Raumkante (axiale Raummoden) zu einer weiteren Verstärkung von Schalldruck und -schnelle entlang dieser Kante. Empirische Untersuchungen zeigen, dass es vor allem bei axialen Moden zu einer Verstärkung von bis zu $20 \mathrm{~dB}$ kommen kann.

Die Überhöhungen von Schalldruck und -schnelle in der Raumkante resultieren folglich aus der Verbindung von Interferenzphänomen und modalem Schallfeld. Diese Überhöhungen haben größere Reibungs- und Wärmeleitungsverluste im porösen Absorbermaterial zur Folge und könnten somit eine Erklärung für die hohe Absorptionswirkung von eben diesem Absorbermaterial in Raumkanten sein.

\section{Methodik}

\subsection{Entwickelter Kantenabsorber}

Ziel der KA-Entwicklung war es, ein leichtes, modulares und flexibles KA-System zu realisieren, das einfach im Aufbau und kosteneffizient ist und eine hohe Breitbandabsorption bei tiefen und tief-mittleren Frequenzen und eine geringe Absorption bei hoch-mittleren und hohen Frequenzen besitzt.

Zunächst wurde ein Gehäuse für den KA entworfen. Anschließend wurden Messungen mit dem Impedanzrohr nach der Messnorm

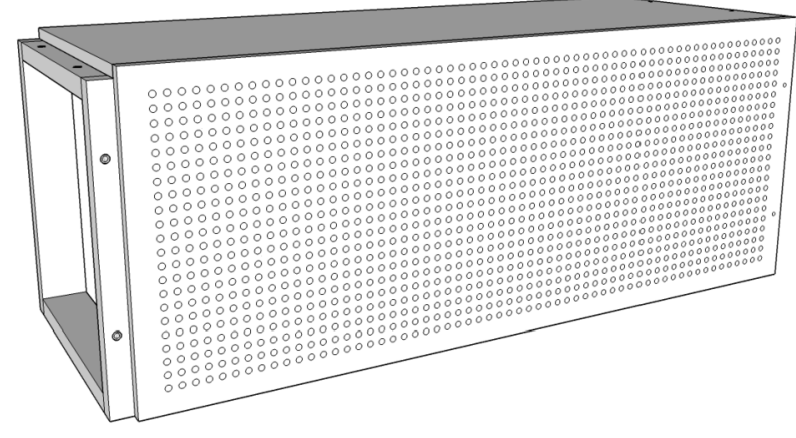

Abb. 6. Modell eines Moduls des entwickelten Gehäusesystems für den KA

ÖNORM EN ISO 10534-2 [16] durchgeführt, um eine optimale Kombination aus absorbierendem porösem Material und einer geeigneten Abdeckung zu finden.

\subsubsection{Modulares KA-System}

Für systematische Untersuchungen wurde ein modulares Gehäusesystem für den KA entwickelt (siehe Abb. 6). Mit diesem System wird eine hohe Montageflexibilität des KA erreicht und der KA kann in Länge, Füllmenge und Abdeckung adaptiert werden. Insgesamt wurden 9 Module mit 1000 mm Länge und 14 Module mit $1500 \mathrm{~mm}$ Länge gefertigt. Alle Module haben einen quadratischen Querschnitt mit einer Tiefe von $b_{K A}=400 \mathrm{~mm}$ und können über ein Stecksystem miteinander verbunden werden. Das modulare Gehäusesystem ermöglicht Messungen verschiedener Konfigurationen des KA (siehe Abschn. 3.3), sowie einfache Montage, Transport und Lagerung. Wie in Abb. 6 dargestellt, ist eine der Abdeckplatten gelocht, damit das Schallfeld in den Absorber eindringen kann. Es wurde festgestellt, dass sich ein Lochverhältnis von $\sigma=20 \%$ optimal für den KA eignet (vgl. Abschn. 3.1.2). Die ungelochte Abdeckplatte sorgt für eine bessere Führung des Schalls im Inneren des KA. Mit $r=\sqrt{2} \cdot b_{\mathrm{KA}}$ und den Gleichungen (8), (9) und (10) kann die Frequenz für ein erwartetes Absorptionsmaximum sowie die obere und untere Grenzfrequenz für effektive Absorption berechnet werden. Es ergeben sich folgende Werte für den entwickelten KA (vgl. GI. (8), (9) und (10)):

$$
\begin{aligned}
f_{\left\langle v_{r}^{2}\right\rangle_{\text {max }}} & =\frac{343 \frac{\mathrm{m}}{\mathrm{s}}}{2 \pi \cdot \sqrt{2} \cdot 0,4 \mathrm{~m}}=97 \mathrm{~Hz} \\
f_{\left\langle v_{r}^{2}\right\rangle}^{\prime \prime} & =\frac{343 \frac{\mathrm{m}}{\mathrm{s}}}{\pi / 2 \cdot \sqrt{2} \cdot 0,4 \mathrm{~m}}=386 \mathrm{~Hz} \\
f_{\left\langle v_{r}^{2}\right\rangle}^{\prime} & =\frac{343 \frac{\mathrm{m}}{\mathrm{s}}}{4 \pi \cdot \sqrt{2} \cdot 0,4 \mathrm{~m}}=48 \mathrm{~Hz}
\end{aligned}
$$

\subsubsection{Materialien}

Insgesamt wurden 170 Messungen verschiedener Materialschichtungen im Impedanzrohr mit Bezug zum modularen KA-System durchgeführt (siehe Abschn. 3.1.1). Basierend auf den Ergebnissen aus den Messungen mit dem Impedanzrohr besteht der KA aus einem absorbierenden porösen Material mit $\Xi \geq 5 \frac{\mathrm{kPa} \cdot \mathrm{s}}{\mathrm{m}^{2}}$, das vollständig mit Molino umhüllt ist. Die Lochplatte für das Gehäuse hat ein Lochverhältnis von $\sigma=20 \%$ und das Gehäuse ist vollständig mit dem absorbierenden porösen Material gefüllt. Das resultierende Absorptionsverhalten ist in Abb. 7 dargestellt.

Natürlich kann der mit dem Impedanzrohr ermittelte Absorptionskoeffizient $\alpha(f)$ des KA nur eine erste Annäherung an sein akustisches Verhalten in einem realen Schallfeld sein, insbesondere wenn 


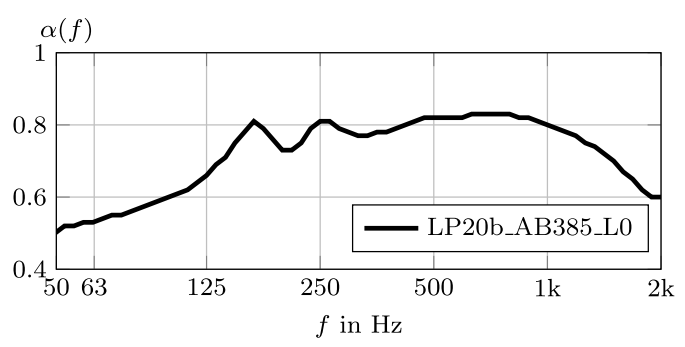

Abb. 7. Absorptionskoeffizient $\alpha(f)$ für die Schichtkonfiguration: Lochplatte mit Lochverhältnis $\sigma=\mathbf{2 0} \%$ und Plattendicke $d_{p}=0,015 \mathrm{~m}$, Lochabdeckung Molino, absorbierendes poröses Material mit Strömungswiderstand $\Xi \geq 5 \frac{\mathrm{kPa} \cdot \mathrm{s}}{\mathrm{m}^{2}}$ und Schichtdicke $d_{a}=0,385 \mathrm{~m}$

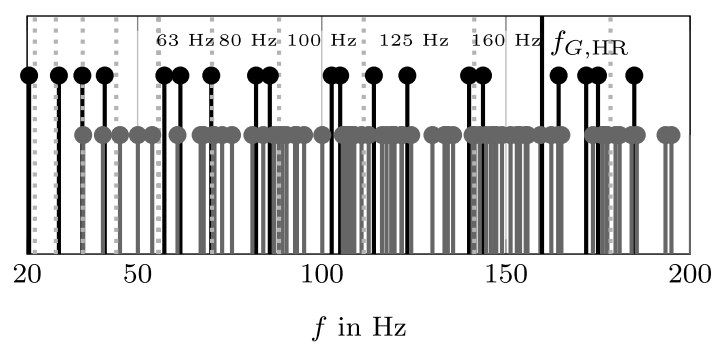

Abb. 8. Verteilung der axialen (๑) und tangentialen (๑) Moden und Grenzfrequenz $f_{G, H R}$ des Hallraums der TU Graz. Vertikale gestrichelte Linien markieren die Grenzfrequenzen des jeweiligen Terzbandes

der KA in einer Raumkante montiert ist. Weiterhin scheint die ParisFormel [17, S. 479] nicht geeignet, um ein einfallswinkelabhängiges $\alpha(\vartheta, \varphi)$ in ein $\alpha_{\text {diff }}$ für diffusen Schalleinfall umzurechnen. Daher ist es erforderlich, Messungen im Hallraum durchzuführen (vgl. Abschn. 3.3).

\subsection{Messaufbau}

Für die Messung der Raumimpulsantworten im Hallraum (Abschn. 3.3) sowie in den Hörsälen (Abschn. 3.4) wurde folgender Lautsprecheraufbau verwendet:

- Norsonic Dodekaeder Lautsprecher Nor276 mit Leistungsverstärker Nor280

- 4 × Lambda Labs CX-1A Zweiwege-Hochleistungskoaxialmonitore mit einem Hochpass bei $140 \mathrm{~Hz}$

- Lambda Labs MF-15A Hochleistungssubwoofer mit einem Tiefpass bei $140 \mathrm{~Hz}$

Alle Lautsprecher wurden simultan bespielt. Die Koaxialmonitore zusammen mit dem Subwoofer erweiterten den Messfrequenzbereich zu hohen und vor allem zu tiefen Frequenzen. Als Anregungssignal wurde ein Sinus-Sweep von $1 \mathrm{~Hz}$ bis $24 \mathrm{kHz}$ mit $f_{S}=48 \mathrm{kHz}$ und einer Länge von $20 \mathrm{~s}$ verwendet.

Der Messmikrofonaufbau bestand aus 6 G.R.A.S. 46AE 1/2" CCP Freifeldmikrofonen, welche an ein PAK MKII Messsystem von MüllerBBM angeschlossen wurden.

\subsection{Hallraum}

Zunächst wurden Messungen des KA im Hallraum am Institut für Bauphysik der Technischen Universität Graz durchgeführt. Der Hallraum entspricht den Anforderungen für Messungen nach ISO 354:2003 [18].
Tab. 1. Untersuchte Hörsäle mit ihren jeweiligen Grundflächen $A_{G}$ und Raumvolumina $V$. Der Hörsaal 2a befindet sich im Bundesrealgymnasium Keplerstraße Graz. Alle anderen Hörsäle befinden sich am Campus Inffeldgasse der Technischen Universität Graz

\begin{tabular}{lcc}
\hline Hörsaal & $A_{\mathrm{G}}$ in $\mathrm{m}^{2}$ & $V$ in $\mathrm{m}^{3}$ \\
\hline 2a & 66 & 243 \\
FSI 1 & 128 & 550 \\
FSI 2 & 62 & 257 \\
i14 & 76 & 265 \\
\hline
\end{tabular}

Mit seinen Abmessungen von 8,35 $\mathrm{m} \times 5,99 \mathrm{~m} \times 4,9 \mathrm{~m}(\mathrm{~L} \times \mathrm{B}$ $\times \mathrm{H}$ ) hat er eine Grundfläche von $A_{G}=50,02 \mathrm{~m}^{2}$, ein Volumen von $V=245,1 \mathrm{~m}^{3}$ und eine Grenzfrequenz [19, S. 344] von

$$
f_{G, H R}=\frac{1000}{\sqrt[3]{V}}=159,8 \mathrm{~Hz} .
$$

Messungen in Frequenzbändern oberhalb von $f_{G, H R}$ können ohne große Einflüsse von einzelnen Raummoden durchgeführt werden. Unterhalb von $f_{G, H R}$ wird das Schallfeld jedoch durch einzelne Moden dominiert. Betrachtet man die modale Verteilung des Hallraums (vgl. Abb. 8), so ist für $f<100 \mathrm{~Hz}$ ein starker Einfluss einzelner Moden auf das Schallfeld zu erwarten. Außerdem gibt es Modenhäufungen bei $70 \mathrm{~Hz}, 83 \mathrm{~Hz}, 105 \mathrm{~Hz}$ und $150 \mathrm{~Hz}$, für welche eine hohe Absorption mit einem KA zu erwarten ist.

\subsection{Hörsäle}

Um die Erkenntnisse aus den Messungen im Hallraum zu verifizieren, wurde eine zusätzliche Messreihe in Hörsälen gemäß Tab. 1 durchgeführt. Die Grundrisse der Hörsäle sind in Abb. 13 und 15 abgebildet.

\section{Ergebnisse}

\subsection{Hallraum}

Die Untersuchungen im Hallraum umfassten den Einfluss des KA auf das Schallfeld bei Positionierung in $x-, y$ - und $z$-Kanten. Weiterhin wurden Kombinationen der Kantenpositionierung sowie Positionierung auf dem Boden und an der Wand vermessen.

Um die Absorptionswirkung des KA besser beurteilen zu können, wird eine äquivalente Absorptionsfläche pro Längeneinheit des KA definiert:

$$
A_{e, l}=\frac{S \cdot \alpha}{l_{\mathrm{KA}}}=\frac{A_{e}}{l_{\mathrm{KA}}}
$$

Hierbei sind die einzelnen Variablen wie folgt definiert:

- S $\quad$... Fläche des KA, die dem Schallfeld zugewandt ist

- $\begin{array}{ccc}\alpha & \ldots & \text { Absorptionskoeffizient }\end{array}$

- $A_{e} \quad \ldots$ äquivalente Absorptionsfläche

- IKA $_{\text {Ka }} \quad \ldots$ Länge des KA

Um die Auswirkung einer entsprechenden Positionierung des KA zu untersuchen, wurden Vergleichsmessungen für Boden- bzw. Wandpositionierung und Kantenpositionierung durchgeführt. Die jeweiligen Positionierungen des KA für die $x-, y$ - und $z$-Kanten im Hallraum sind in Abb. 9 dargestellt. Aus Gründen der Vergleichbarkeit erfolgte die Positionierung des KA in z-Richtung in zwei Kanten an derselben Wand des Hallraums. Die resultierenden KA-Längen/Flächen sind 7,5 m/6,32 $\mathrm{m}^{2}, 5,5 \mathrm{~m} / 4,72 \mathrm{~m}^{2}$ und $7 \mathrm{~m} / 5,92 \mathrm{~m}^{2}$ für $x$-, $y$ - und z-Richtung

Abbildung 10 zeigt die äquivalente Absorptionsfläche pro Längeneinheit $A_{e, l}$ für die Positionierung des KA in den drei Richtungen 


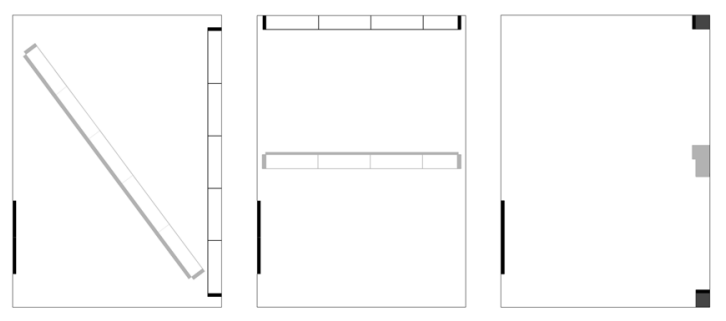

Abb. 9. Unterschiedliche KA-Positionierung entlang der $x-, y$ - und $z$-Kanten ( $a, b$ und $c$; schwarze Elemente) des Hallraums sowie die entsprechende KA-Positionierung auf dem Boden ( $a$ und $b$ ) und an der Wand (c) für die Vergleichsmessungen (graue Elemente)

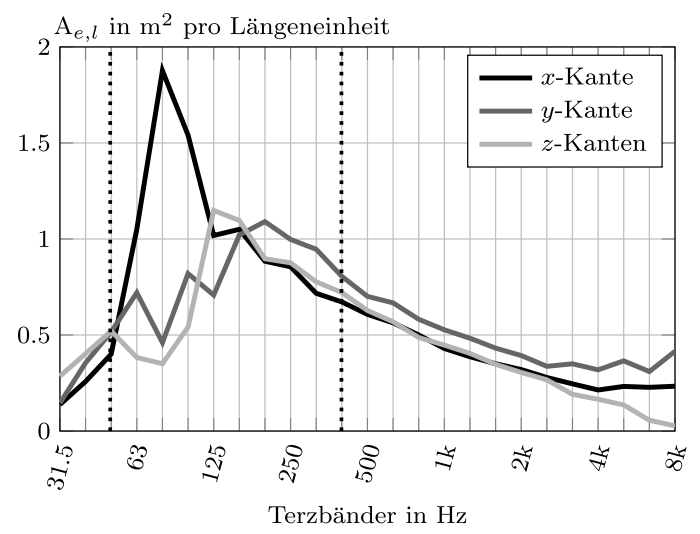

Abb. 10. Äquivalente Absorptionsfläche pro Längeneinheit $A_{e, l}$ in Terzbändern für KA-Positionierung in $\boldsymbol{x}-, \boldsymbol{y}$ - und $\boldsymbol{z}$-Kanten des Hallraums gemäß Abb. 9. Vertikale gestrichelte Linien $\left(f_{\left\langle v_{r}^{2}\right\rangle}^{\prime}\right.$ und $\left.f_{\left\langle v_{r}^{2}\right\rangle}^{\prime \prime}\right)$ markieren die erwartete Bandbreite für die effektive Absorption des KA (vgl. Abschn. 3.1.1)

der Raumkanten des Hallraums (vgl. Abb. 9). Es ist ersichtlich, dass das Maximum $A_{e, l}$ für jede KA-Positionierung bei unterschiedlichen Frequenzen $(80 \mathrm{~Hz}, 125 \mathrm{~Hz}$ und $200 \mathrm{~Hz}$ ) erreicht wird. Der Hauptbeitrag zur Absorption für die jeweilige KA-Positionierung liegt aber im Frequenzbereich der effektiven Absorption. Für Frequenzen oberhalb von $f_{\left\langle v_{r}^{2}\right\rangle}^{\prime \prime}$ gleicht sich die Absorption der KA-Konfigurationen aneinander an. Zum besseren Verständnis der Auswirkung der Positionierung der KA in den Raumkanten wurden außerdem die gleichen Absorberkonfigurationen für die Boden-Mandpositionierung gemessen (vgl. Abb. 9, graue Elemente). Anschließend wurde die resultierende äquivalente Absorptionsfläche pro Längeneinheit für die Boden-Mandpositionierung $\hat{A}_{e, l}$ verwendet, um die Differenz $\Delta A_{e, l}$ zwischen Kanten- und Flächenpositionierung des KA zu berechnen (13). Mit diesem Verfahren kann der relative Gewinn von $A_{e, l}$ durch die Positionierung des KA in den Raumkanten gezeigt werden.

$$
\Delta A_{e, l}=A_{e, l}-\hat{A}_{e, l}
$$

Die Ergebnisse des Verfahrens sind in Abb. 11 aufgetragen. Es ist ersichtlich, dass das höchste $\Delta A_{e, l}$ für die jeweilige KA-Positionierung wieder in der erwarteten Bandbreite für die effektive Absorption zu finden ist. Die Frequenzen für den maximalen Gewinn an $A_{e, l}$ sind abhängig von der Positionierungsrichtung des KA und die Frequenzen stimmen mit denen der Maxima aus Abb. 10 überein. Obwohl es für die $z$-Kanten ein weniger ausgeprägtes Absorptionsmaximum im Vergleich zur $x$-Kantenpositionierung des KA gibt (vgl. Abb. 10),

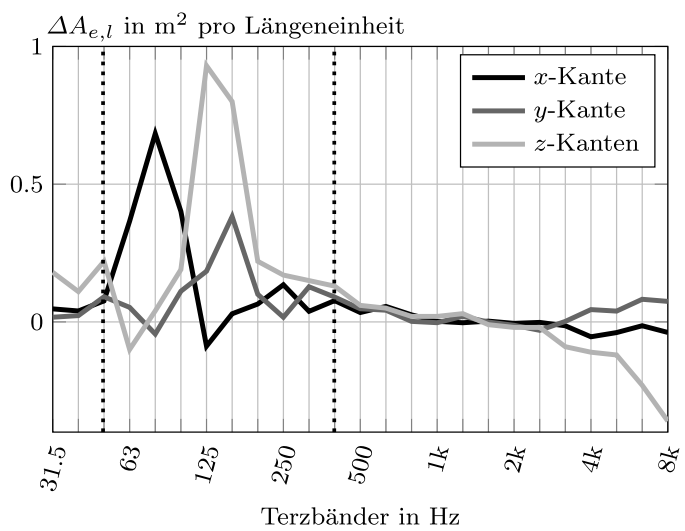

Abb. 11. Differenz der äquivalenten Absorptionsfläche pro Längeneinheit $\Delta A_{e, l}$ zwischen Kanten- und Oberflächenpositionierung des $K A$. Vertikale gestrichelte Linien $\left(f_{\left\langle v_{r}^{2}\right\rangle}^{\prime}\right.$ und $\left.f_{\left\langle v_{r}^{2}\right\rangle}^{\prime \prime}\right)$ markieren die erwartete Bandbreite für die effektive Absorption des KA (vgl. Abschn. 3.1.1)

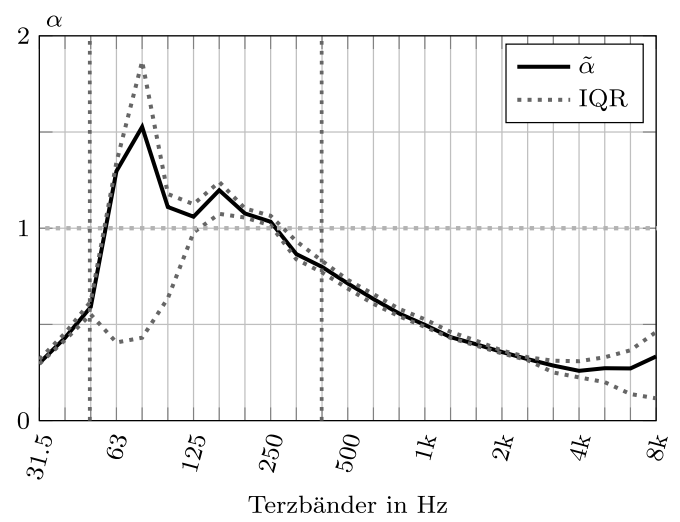

Abb. 12. Medianwerte des Absorptionskoeffizienten $\tilde{\alpha}$ für Messungen mit verschiedenen KA-Konfigurationen mit Interquartilsabständen (IQR) in Terzbändern. Vertikale gestrichelte Linien $\left(f_{\left\langle v_{r}^{2}\right\rangle}^{\prime}\right.$ und $\left.f_{\left\langle v_{r}^{2}\right\rangle}^{\prime \prime}\right)$ markieren die erwartete Bandbreite für die effektive Absorption des KA (vgl. Abschn. 3.1.1)

ist die relative Verstärkung zur jeweiligen Flächenpositionierung ausgeprägter.

Weitere Konfigurationen des KA, darunter eine Positionierung in $x-, y$ - und $z$-Kanten gleichzeitig, eine Positionierung mit unterschiedlicher Anzahl von KA-Türmen in den $z$-Kanten sowie eine gürtelartige Positionierung mit und ohne KA-Türme in den z-Kanten, wurden gemessen. Eine weitere detaillierte Darstellung der Ergebnisse würde den Rahmen dieses Beitrags sprengen. Für all diese Messungen kann jedoch der Medianwert der Absorptionskoeffizienten $\tilde{\alpha}$ mit dem jeweiligen Interquartilsabstand (IQR) aufgetragen werden (vgl. Abb. 12). Für $f_{\left\langle v_{r}^{2}\right\rangle}^{\prime}<f<f_{\left\langle v_{r}^{2}\right\rangle}^{\prime \prime}$ kann die erwartete effektive Absorption $(\alpha \geq 1)$ weitgehend bestätigt werden. Der große IQR für $\alpha$ zwischen 63 und $100 \mathrm{~Hz}$ verdeutlicht, dass die resultierende Absorption stark von der Positionierung des KA abhängig ist. Weniger positionsabhängige Variationen von $\alpha$ liegen für $f>f_{\left\langle v_{2}^{2}\right\rangle}^{\prime \prime}$ vor, wo die Absorption hauptsächlich durch das akustische Verhalten des porösen Absorbermaterials selbst bestimmt wird. 

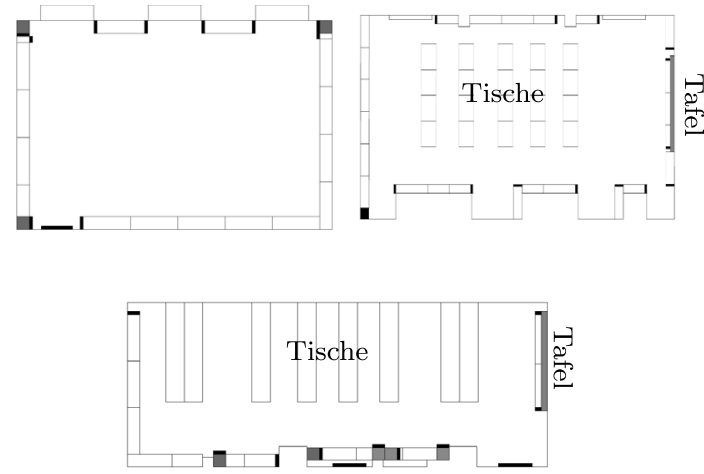

Abb. 13. Grundrisse der Hörsäle (a) 2a, (b) FSI1 und (c) i14 mit den effektivsten KA-Konfigurationen

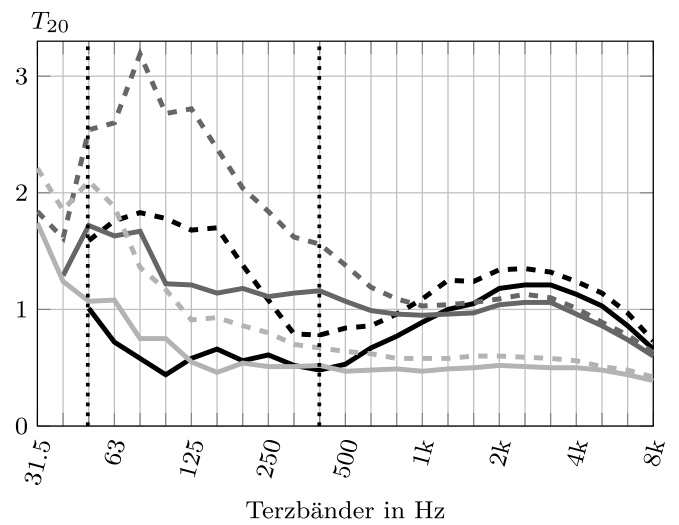

Abb. 14. Ausgangswert (- - -) und resultierender Wert der $T_{20}(-)$ für Hörsäle 2a $(--,-$,$) , FSI1 (--,-)$ und i14 $(--,-)$ in Terzbändern Vertikale gestrichelte Linien $\left(f_{\left\langle v_{r}^{2}\right\rangle}^{\prime}\right.$ und $\left.f_{\left\langle v_{r}^{2}\right\rangle}^{\prime \prime}\right)$ markieren die erwartete Bandbreite für die effektive Absorption des KA (vgl. Abschn. 3.1.1)

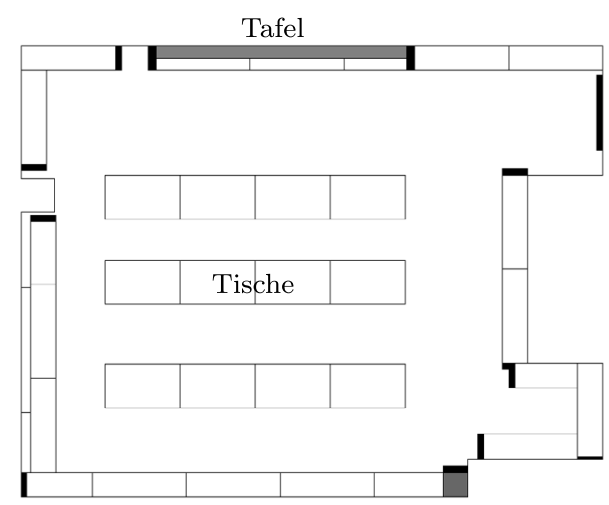

Abb. 15. Grundriss des Hörsaals FSI2 mit effektivster KA-Konfiguration

\subsection{Hörsäle}

In jedem Hörsaal aus Tab. 1 wurden mehrere KA-Konfigurationen vermessen. Aus Gründen der Kompaktheit sind in den Abbildungen 13 und 15 nur jene KA-Konfigurationen mit der effektivsten Absorption dargestellt. Die jeweiligen Nachhallzeiten $T_{20}$ für die Hörsäle mit und ohne diese KA-Konfigurationen sind in Abb. 14 und 16 dargestellt. Auch hier zeigen die Ergebnisse, dass für niedrige Frequen-

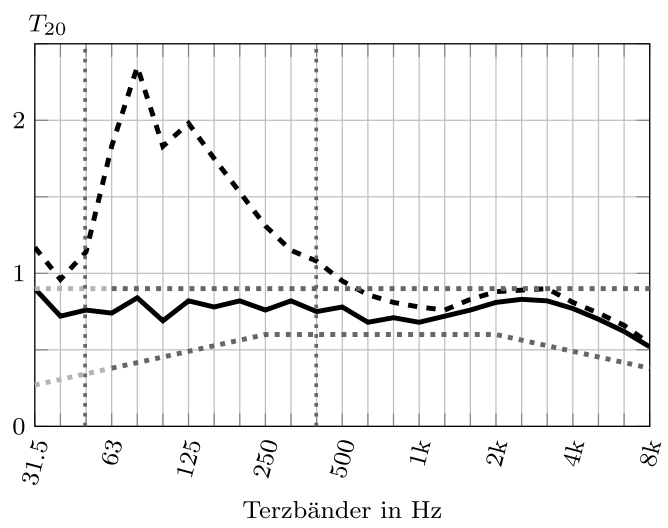

Abb. 16. Ausgangswert (- - -) und resultierender Wert für $T_{20}(-)$ für Hörsaal FSI2. Horizontale gestrichelte Linien markieren die minimalen und maximalen Werte für $T_{20}$ für den Anwendungsfall "Sprache" gemäß ÖNORM B 8115-3 [20]. Vertikale gestrichelte Linien $\left(f_{\left\langle v_{r}^{2}\right\rangle}^{\prime}\right.$ und $\left.f_{\left\langle v_{r}^{2}\right\rangle}^{\prime \prime}\right)$ markieren die erwartete Bandbreite für die effektive Absorption des KA (vgl. Abschn. 3.1.1)

zen $\left(f_{\left\langle v^{2}\right\rangle}^{\prime}<f<f_{\left(v_{2}^{2}\right\rangle}^{\prime \prime}\right)$, bei denen ein modales Schallfeld vorliegt, mit dem KA eine hervorragende Absorption erzielt werden kann. Raummoden, unabhängig von ihrer Frequenz, werden effizient bedämpft. Daher ist der Ausdruck Modenbremse sehr gut geeignet um das akustische Verhalten des KA zu beschreiben. Ist die Modenverteilung in einem bestimmten Terzband sehr dicht, wird eine Reduzierung der Nachhallzeit $T_{20}$ in diesem Band erreicht. Im Mittel kann die Nachhallzeit $T_{20}$ für alle Hörsäle um $47 \%\left(\Delta \overline{T_{20}}=0,83 \mathrm{~s}\right)$ in der erwarteten Bandbreite für effiziente Absorption reduziert werden. Die größten Reduktionen von $T_{20}$ können für Hörsaal $2 \mathrm{a}$ im $100 \mathrm{~Hz}$-Band $\left(75 \%\right.$ bzw. $\left.\Delta T_{20}=1,34 \mathrm{~s}\right)$ und für Hörsaal FSI1 im $80 \mathrm{~Hz}-$ Band ( $48 \%$ bzw. $\Delta T_{20}=1,52 \mathrm{~s}$ ) erreicht werden. Für Frequenzen $f>1 \mathrm{kHz}$ ist der Effekt der KA marginal. Für den Hörsaal FSI2 kann eine starke Reduktion von $T_{20}$ für die Terzbänder von 63 bis $200 \mathrm{~Hz}$ mit einer mittleren Differenz $\Delta \overline{T_{20}}=1,13 \mathrm{~s}$ beobachtet werden. Durch den Einsatz dieser KA-Konfiguration im FSI2 können die Anforderungen an die Nachhallzeit gemäß ÖNORM B 8115-3 für alle Terzbänder erreicht werden. Nach dem Einbringen des KA beträgt die mittlere Nachhallzeit $\overline{T_{20}}=0,75 \mathrm{~s}$ mit einer Standardabweichung von $\sigma_{T_{20}}=0,08 \mathrm{~s}$.

\section{Diskussion}

Die Erkenntnisse aus den Messungen im Hallraum können die Untersuchungen aus Abschn. 2.1 und 2.2 bestätigen. Die Modenhäufungen in den Terzbändern von 63 bis $160 \mathrm{~Hz}$ dominieren das Schallfeld unterhalb der Grenzfrequenz $f_{G, H R}$ (vgl. Abb. 8). Sie liegen in der erwarteten Bandbreite für die effektive Absorption des $\mathrm{KA}, f_{\left\langle v_{r}^{2}\right\rangle}^{\prime}<f<f_{\left\langle v_{c}^{2}\right\rangle}^{\prime \prime}$, und können somit effektiv bedämpft werden. Natürlich hängt die Dämpfungseffizienz auch stark von der Einbausituation des KA ab. Jedoch werden sowohl axiale als auch tangentiale Moden, die in der jeweiligen Raumkante auftreten und in der erwarteten Bandbreite für effektive Absorption liegen, unabhängig von ihrer Frequenz stark abgeschwächt. Unterhalb der unteren Grenzfrequenz für effektive Absorption $f_{\left\langle v_{r}^{2}\right\rangle}^{\prime}$ ist der Einfluss des KA auf das Schallfeld nicht mehr so stark gegeben (Abb. 12). Oberhalb von $f_{\left\langle v_{r}^{2}\right\rangle}^{\prime \prime}$ ist keine hohe kinetische und potentielle Energie in der Raumkante mehr zu erwarten und das Absorptionsverhalten des KA wird hauptsächlich durch das poröse Material selbst bestimmt (Abb. 12). $f_{\left\langle v_{r}^{2}\right\rangle}^{\prime}, f_{\left\langle v_{r}^{2}\right\rangle}^{\prime \prime}$ sowie $f_{\left\langle v_{r}^{2}\right\rangle \max }$ sind abhängig von der Einbautie- 
fe des KA. Für den entwickelten KA liegt $f_{\left\langle v_{r}^{2}\right\rangle \text { max }}$ im $80 \mathrm{~Hz}$ Terzband und die in diesem Band auftretenden Überhöhungen der Nachhallzeit können bei geeigneter Positionierung des KA drastisch vermindert werden (Abb. 14 und 16).

Die Ergebnisse der Messungen in den Hörsälen zeigen, dass eine gürtelartige Anordnung der KA zusammen mit KA-Türmen eine sehr gute Absorption erzielen können (Abb. 13 und 14). Es erscheint sinnvoll, dass mindestens eine Kante in jeder Dimension des Raumes mit einem KA besetzt ist. Eine spezifische Abstimmung des KA für bestimmte Modenfrequenzen ist nicht erforderlich. Weiterhin kann mit einer relativ kleinen Absorberoberfläche eine gute Schallabsorption bei niedrigen Frequenzen erreicht werden. Die hervorragenden Ergebnisse durch Einbringen des KA in den FSI2 führte zu einer akustischen Sanierung des Hörsaals mit eben diesem. Durch die Montage der gleichen KA-Konfiguration (vgl. Abb. 15) unterhalb der Raumdecke konnte ein unauffälliges Absorbersystem realisiert werden, das die Sprachverständlichkeit im Raum empfindlich verbessert.

Zur Simulation der Absorptionseigenschaften des KA in einfachen Raumgeometrien könnten Gewichte für jedes Terzband $w_{M}(f)$ in Abhängigkeit der Verteilung der Raummoden eingeführt werden. Diese Gewichte könnten zusammen mit dem Verhältnis zwischen bedeckten und unbedeckten Kanten durch den KA in jeder Raumdimension $w_{l}=I_{x_{i}, K A} / l_{x_{i}}$ und dem Absorptionskoeffizienten $\alpha(f)$ für die jeweilige Absorberkonstruktion, z.B. ermittelt im Impedanzrohr (vgl. Abschn. 3.1.2), zur Abschätzung der Wirkung des KA auf das modale Schallfeld verwendet werden. Um die Bandbreite der effektiven Absorption und des Absorptionsmaximums des KA zu ermitteln, erscheint die Verwendung seiner Tiefenabmessung sinnvoll (vgl. Abb. 4).

\section{Konklusion}

Durch die systematische Untersuchung der Absorptionscharakteristik des KA konnte nachgewiesen werden, dass eine Verbesserung der Raumakustik und insbesondere der breitbandigen Raummodenunterdrückung durch den Einsatz des Kantenabsorbers möglich ist. Durch Reibungs- sowie Wärmeleitungsverluste im porösen Absorbermaterial des KA kann dem modalen Schallfeld, welches auf Grund der Interferenzerscheinungen gerade entlang der Raumkanten ausgeprägte Schalldruck- und Schallschnelleüberhöhungen aufweist, effektiv Schallenergie entzogen werden. Somit ergibt sich eine automatisch erhöhte Absorption von ausgeprägteren Raummoden. Durch diesen Effekt wirkt der KA wie eine breitbandige "Modenbremse", und zwar umso besser, je stärker die Moden ausgeprägt sind.

Aus theoretischen Untersuchungen konnte herausgefunden werden, dass das Zusammenspiel von Schalldruck- und Schallschnelleanstieg in einer Raumkante und die Verteilung dieser beiden akustischen Feldgrößen in einem modalen Schallfeld der Grund für eine hohe Absorptionswirkung des KA sein könnte. Dabei konnte aus dem Interferenzmuster der Schallschnelle $v$ in einer Raumkante ein zu erwartendes Absorptionsmaximum $f_{\left\langle v_{r}^{2}\right\rangle \text { max }}$ sowie eine Bandbreite für die effektive Absorption $\left.\left(f_{\left\langle v_{r}^{2}\right\rangle}^{\prime}\right\rangle^{\prime}, f_{\left\langle v_{r}^{2}\right\rangle}^{\prime \prime}\right)$ in Abhängigkeit vom Abstand $r$ abgeleitet werden.

Die berechneten Werte für $f_{\left\langle v_{r}^{2}\right\rangle \max }, f_{\left\langle v_{r}^{2}\right\rangle}^{\prime}$ und $f_{\left\langle v_{r}^{2}\right\rangle}^{\prime \prime}$ konnten durch Messungen im Hallraum und in Hörsälen bestätigt werden. Das Absorptionsmaximum eines KA wird jedoch nicht nur durch seine Tiefenabmessung bestimmt, sondern auch durch die Positionierung des $\mathrm{KA}$ in einer bestimmten Raumgeometrie und der daraus resultierenden Wechselwirkung mit dem modalen Schallfeld. Im Allgemeinen ist die längste Raumkante, entlang derer sich die tiefstmöglichen Raummoden ausbilden können, die bevorzugte Einbauposition eines KA. Eine breitbandige Absorption kann durch die Montage des
KA entlang mindestens einer Kante jeder Raumdimension erreicht werden.

In der Praxis ist die Kenntnis der genauen Modenstruktur eines Raumes nicht erforderlich, da durch die Positionierung in den Raumkanten eine ausreichende Wechselwirkung des KA mit dem modalen Schallfeld erzielt wird. Eine spezifische Abstimmung des KA ist aufgrund seiner Bandbreite mit effektiver Absorption nicht erforderlich. Generell verbessert ein hohes Verhältnis zwischen bedeckten und unbedeckten Kanten durch den KA die Bedämpfung der Raummoden.

Für die weitere Entwicklung eines Simulationswerkzeugs für Kantenabsorber in einfachen Raumgeometrien könnte ein erster Ansatz Kenntnisse über die Verteilung der Raummodenfrequenzen enthalten. Berechnete Gewichte $w_{M}(f)$ für die Dichte der Raummodenverteilung in Terzbändern zusammen mit dem Absorptionskoeffizienten $\alpha(f)$ für den KA und $w_{\text {l, }}$ dem Verhältnis zwischen der vom KA bedeckten und unbedeckten Kantenlänge in jeder Raumdimension, scheinen sinnvolle Parameter für eine Simulation der akustischen Eigenschaften des KA zu sein.

Funding Note Open access funding provided by Graz University of Technology.

Hinweis des Verlags Der Verlag bleibt in Hinblick auf geografische Zuordnungen und Gebietsbezeichnungen in veröffentlichten Karten und Institutsadressen neutral.

Open Access Dieser Artikel wird unter der Creative Commons Namensnennung 4.0 International Lizenz veröffentlicht, welche die Nutzung, Vervielfältigung, Bearbeitung, Verbreitung und Wiedergabe in jeglichem Medium und Format erlaubt, sofern Sie den/die ursprünglichen Autor(en) und die Quelle ordnungsgemäß nennen, einen Link zur Creative Commons Lizenz beifügen und angeben, ob Änderungen vorgenommen wurden. Die in diesem Artikel enthaltenen Bilder und sonstiges Drittmaterial unterliegen ebenfalls der genannten Creative Commons Lizenz, sofern sich aus der Abbildungslegende nichts anderes ergibt. Sofern das betreffende Material nicht unter der genannten Creative Commons Lizenz steht und die betreffende Handlung nicht nach gesetzlichen Vorschriften erlaubt ist, ist für die oben aufgeführten Weiterverwendungen des Materials die Einwilligung des jeweiligen Rechteinhabers einzuholen. Weitere Details zur Lizenz entnehmen Sie bitte der Lizenzinformation auf http://creativecommons.org/licenses/by/4.0/deed.de.

\section{Literatur}

1. Wegel, R., Lane, C. (1924): The auditory masking of one pure tone by another and its probable relation to the dynamics of the inner ear. Phys. Rev., 23(2), 266-285.

2. Tobias, J. V. (1977): Low-frequency masking patterns. J. Acoust. Soc. Am., 61(2), $571-575$

3. Gelfand, S. A. (2010): Hearing: an introduction to psychological and physiological acoustics. London: Informa UK.

4. Kuttruff, H. (1994): Taschenbuch der Technischen Akustik. Berlin: Springer.

5. Fuchs, H. V. (2011): Funktionelle Akustik - Die Nachhall-Charakteristik des Raumes als Basis für seine Nutzbarkeit: Teil 4: Bauliche Maßnahmen in Räumen für musikalische und schalltechnische Nutzungen. Bauphysik, 33(4), 195-206.

6. Fuchs, H. V., Lamprecht, J., Zha, X. (2011): Zur Steigerung der Wirkung passiver Absorber: Schall in Raumkanten schlucken. Gesund.-Ing., 132(5), 240-252.

7. Fuchs, H. V., Graber, G., Hetz, S., Kordesch, J., Balint, J. (2017): Harte Kanten für klare Worte. Trockenbau Akust., 34(6), 70-73.

8. Waterhouse, R. (1955): Interference patterns in reverberant sound fields. J. Acoust. Soc. Am., 27, 247-259.

9. Wefers, F., Stienen, J., Pelzer, S., Vorländer, M. (2014): Interactive acoustic environments using distributed room acoustic simulations. In Proceedings of the EAA joint symposium on auralization and ambisonics, Apr. 2014, Berlin (S. 48-55).

10. COMSOL AB, COMSOL Acoustic Module. Website [Online]. Available https://www. comsol.com/acoustics-module.

11. Reisinger, D. (2019): Entwicklung und Messung von Kantenabsorbern. Master Thesis, Graz University of Technology.

12. Kurz, E., Reisinger, D., Graber, G., Weselak, W. (2020): The edge absorber as a "Modal Brake". In Conference proceedings e-forum Acusticum 2020, Dec. 2020.

13. Maa, D. (1940): Non-uniform acoustical boundaries in rectangular rooms. J. Acoust. Soc. Am., 12(1), 39-52. 
14. Kuhl, W. (1960): Der Einfluss der Kanten auf die Schallabsorption poröser Materialien Acustica, 10, 264-276.

15. Plagg, S., Pobitzer, M. (Jul. 2011): Eigenfrequenzen - Eigenmoden, Resonanzfrequenzen - Raummoden. Bachelor Thesis, Graz University of Technology.

16. ÖNORM EN ISO 10534-2:1998 (1998): Akustik - Bestimmung des Schallabsorptionsgrades und der Impedanz in Impedanzrohren - Teil 2: Verfahren mit Übertragungsfunktion, Österreichisches Normungsinstitut Std.

\section{Autoren}

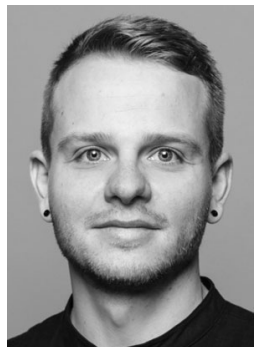

\section{Eric Kurz}

schloss 2018 das interuniversitäre ingenieurwissenschaftliche Masterstudium Elektrotechnik-Toningenieur an der Technischen Universität Graz, Österreich, und der Universität für Musik und darstellende Kunst Graz, Österreich, mit dem akademischen Grad Dipl.Ing. ab. Von März 2019 bis August 2019 war er als wissenschaftlicher Mitarbeiter am Institut für Technische Akustik der RheinischWestfälischen Technischen Hochschule Aachen, Deutschland, tätig Seit September 2019 ist er Universitätsassistent am Institut für Signalverarbeitung und Sprachkommunikation der Technischen Universität Graz, Österreich, im Forschungsbereich der Raumakustik. Seine aktuellen Forschungsinteressen umfassen modale sowie spatial und direktional hochaufgelöste Schallfeldanalyse und räumliche Audiowiedergabe und -signalverarbeitung.

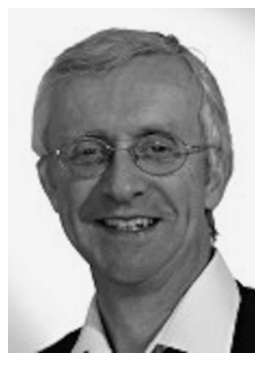

\section{Gerhard Graber}

absolvierte 1981 das interuniversitäre Studium Elektrotechnik-Toningenieur an der Technischen Universität Graz und der damaligen Hochschule für Musik und darstellende Kunst Graz, promovierte 1989 und habilitierte sich 2000 im Fach Audiotechnik. Er ist Universitätsdozent am Institut für Signalverarbeitung und Sprachkommunikation der Technischen Universität Graz und lehrt und forscht
17. Fouad, N. A. (2009): Bauphysik-Kalender 2009: Schallschutz und Akustik (Bd. 9). New York: Wiley.

18. ISO 354:2003 (2003): Acoustics - Measurement of sound absorption in a reverberation room. International Organization for Standardization Std.

19. Henn, H., Sinambari, G. R., Fallen, M. (2008): Ingenieursakustik. Wiesbaden: Vieweg+Teubner/GWV Fachverlage GmbH.

20. ÖNORM B 8115-3:2005 (2005): Schallschutz und Raumakustik im Hochbau - Teil 3 : Raumakustik, Österreichisches Normungsinstitut Std. in Bereich Audiotechnik und Akustik. Im Bereich der Raumakustik gilt sein Interesse der Klassenraumakustik sowie der Wechselwirkung von modalem Schallfeld und Absorber bei tiefen Frequenzen, was zu einer ausführlichen Beschäftigung mit dem Kantenabsorber führt. Als Studiendekan ist er zuständig für das interuniversitäre Elektrotechnik-Toningenieurstudium, das gemeinsam von der Technischen Universität Graz und der Universität für Musik und darstellende Kunst in Graz angeboten wird.

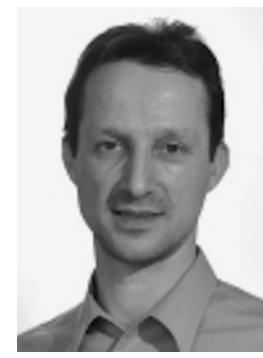

\section{Werner Weselak}

absolvierte sowohl das Diplomstudium als auch das Doktoratsstudium im Fach Elektrotechnik-Toningenieur/Akustik an der Technischen Universität Graz. Er hat mehrere Jahre Industrieerfahrung auf dem Gebiet der Akustik der Automobile und Haushaltsgeräte und Erfahrung als Akustikberater für die Industrie im Bereich der Technischen Akustik. Aktuell ist er tätig als Senior Lecturer in der Akustik-Gruppe des Instituts für Signalverarbeitung und Sprachkommunikation (SPSC) der Technischen Universität Graz. Sein universitärer Schwerpunkt liegt in der Lehre der Fachgebiete Elektroakustik, Akustische Messtechnik und Schallabsorber jeweils im Anwendungsgebiet der Technischen Akustik. 